\title{
An Improved Method to Track Changes of Candidatus Liberibacter asiaticus Titer in HLB-affected Citrus Trees
}

\author{
Ed Etxeberria ${ }^{1}$ and Pedro Gonzalez \\ Department of Horticultural Sciences, University of Florida, Citrus Research \\ and Education Center, Lake Alfred, FL 33850
}

\section{Ariel Singerman and Timothy Ebert \\ Department of Agricultural Economics, University of Florida, Citrus Research and Education Center, Lake Alfred, FL 33850}

Additional index words. Citrus, citrus greening, HLB testing

\begin{abstract}
Monitoring the health of Huanglongbing-affected citrus trees by following changes in leaf Candidatus Liberibacter asiaticus (CLas) titer has an inherent element of imprecision because $C$ Las titer varies considerably within the tree canopy and with calendar seasons. In addition, the destructive sampling method used to determine $C$ Las titer entails a different set of leaves per sampling period adding to the inconsistency and inexactitude of the results. To overcome these ambiguities and to reduce the numerical variability between samples, we developed an experimental method that analyzes portions of the same treated leaves for up to four sampling periods. By assaying subsamples of adjacent locations of the same leaf, random variability was significantly reduced, and comparative analysis can be carried out with greater precision.
\end{abstract}

Huanglongbing (HLB or citrus greening) is the most destructive disease of citrus worldwide, causing extensive production loss in terms of fruit yield and juice quality (Gottwald, 2010). HLB is associated with different strains of the bacterium Candidatus Liberibacter. Of the three species identified so far, only CLas has been found in North America. CLas is vectored by the phloemfeeding psyllid Diaphorina citri (Halbert and Manjunath, 2004) and transmitted into the phloem of citrus leaves during the feeding process. Once inside the leaf, CLas moves systemically throughout the plant (Aritua et al., 2013; Tatineni et al., 2008) initiating a cascade of physiological and metabolic changes that culminate in reduced vigor, diminished production, and ultimately tree death (Aritua et al., 2013; Etxeberria et al., 2009). The systemic bacterial disease has become established in four continents, threatening the survival of citrus production in many countries. In the US state of Florida alone, the disease has been a major cause of the more than $60 \%$ decline in production and cultivated acreage since its was first found in 2005 (U.S. Department of Agriculture, NASS, Florida Citrus Statistics, 2018).

In Florida and elsewhere, major efforts are being undertaken and resources committed to find a solution to this devastating disease. Until a long-term genetic resistance/tolerance solution to this problem is

Received for publication 11 Mar. 2019. Accepted for publication 28 Apr. 2019.

'Corresponding author. E-mail: eetxeber@ufl.edu. uncovered, in the short term, attempts are also being made to ameliorate the effects of HLB in existing trees. To improve the health of HLB-affected citrus trees, treatments such as antimicrobial applications (Zhang et al., 2014), foliar imidacloprid-induced system acquired resistance (Graham et al., 2008), and nutritional sprays (Roberts et al., 2014) are being tested, among other methods. The infectious status of citrus trees is determined on a CLas titer scale where DNA amplification cycle threshold $(\mathrm{Ct})$ number inversely reflects the level of bacterial infection. $\mathrm{Ct}$ levels exceeding background levels $(\leq 35)$ are considered indicative of HLB presence, with higher levels of infection represented by descending $\mathrm{Ct}$ numerical values. Nevertheless, monitoring the health of field-grown, HLB-affected citrus trees by following changes in leaf CLas-DNA titer has an inherent element of imprecision because $C$ Las titer varies considerably within the citrus canopy (Irey et al., 2006; Li et al., 2009; Louzada et al., 2016; McLean, 1970; Stover et al., 2010; Tatineni et al., 2008; Texeira et al., 2008), and the destructive sampling method used to determine CLas titer ( $\mathrm{Li}$ et al., 2007; Stover and McCollum, 2011) entails a different set of leaves per sampling period. In addition, a significant temporal fluctuation (Irey et al., 2011) adds to the inconsistency and inexactitude of the results.

To overcome the ambiguities in CLas titer determinations by using a different set of leaves for every test and to lower the numerical variability between samples, we developed a methodology that analyzes portions of the same treated leaves for up to four sam- pling periods. We hypothesized that by assaying subsamples of adjacent locations of the same leaf, random variability is significantly reduced, and comparative analysis can be carried out with greater precision.

\section{Materials and Methods}

Tissue sampling. Leaf samples for CLas DNA determinations by quantitative polymerase chain reaction (qPCR) were obtained by using a paper hole puncher able to dissect $27-\mathrm{mm}^{2}$ discs. A total of six discs (one disc per leaf) constituted a sample collectively weighing $\approx 100 \mathrm{mg}$. The paper hole punchers were cleaned with $90 \%$ ethanol between each leaf sample. When necessary, samples were stored at $-20{ }^{\circ} \mathrm{C}$. In our experiments, the first leaf disc sample was excised at the midrib toward the center of the leaf, leaving sufficient tissue for the removal of subsequent samples toward the petiole. This is significant because initially removing midrib tissue too close to the petiole could result in leaf yellowing, likely the result of water and photoassimilate imbalance. Ordinarily, titer measurements are performed by tissue grinding and plating of the bacteria for cell counts. However, the bacterium at issue has not been cultured; therefore, we used semiquantitative PCR instead as an estimate instead.

DNA isolation. For isolation of CLas DNA, leaf discs were placed in an Ependorf tube, frozen in liquid nitrogen, and homogenized with one metal ball using a TissueLyser II (TL Qiagen, Valencia, CA). The pulverized tissue was extracted for DNA using the DNeasy Plant Mini Kit (Qiagen, Valencia, CA) following the manufacturer's instructions. Yield and purity of DNA samples were estimated by measuring $\mathrm{OD}_{260}$ and $\mathrm{OD}_{260 / 280}$, respectively, with a NanoDrop Microplate Reader Spectrophotometer Epoch (BioTek Instruments, Inc., Winooski, VT). The final concentration of DNA in all samples was diluted to $4 \mathrm{ng} / \mu \mathrm{L}$ for all samples.

$q P C R$ analysis. Assays were performed on an Applied Biosystems 7500 PCR instrument (Life Technologies, Carlsbad, CA). The TaqMan Universal PCR Master Mix (Life Technologies) was used for the DNA assay. DNA samples were analyzed with degenerate genus-specific $(r p o \mathrm{~B})$ primer/probe sets (Ananthakrishnan et al., 2013). The final concentration of qPCR mix contained $1 \mathrm{X}$ TaqMan Universal PCR Master Mix (10 $\mu \mathrm{L}$ ), forward and reverse primers $2 \mu \mathrm{L}$ each (600 nM each), probe $1 \mu \mathrm{L}(300 \mathrm{nM}), 5 \mu \mathrm{L}$ of $4 \mathrm{ng} / \mu \mathrm{L}$ DNA sample (20 ng total), and nuclease free water for a total of $20 \mu \mathrm{L}$. The qPCR assay consisted of 2 min incubation at $50{ }^{\circ} \mathrm{C}$ followed by $10 \mathrm{~min}$ incubation at $95{ }^{\circ} \mathrm{C}$ and 40 cycles at $95{ }^{\circ} \mathrm{C}$ for $15 \mathrm{~s}$ and $60{ }^{\circ} \mathrm{C}$ for $1 \mathrm{~min}$, respectively. Data were analyzed with the Applied Biosystems software (version 1.4.0) and expressed as the DNA amplification cycle threshold $(\mathrm{Ct})$ number. $\mathrm{Ct}$ values were calculated based on a previously determined standard curve according to Ananthakrishnan et al. (2013). We assigned qPCR Ct values $\leq 35$ as positive 
for 16s rRNA CLas infection. At no time were $\mathrm{Ct}$ values indicative of $C$ Las absence $(>35)$ obtained.

Variability test. Six random HLBsymptomatic leaves from a field grown 'Valencia' sweet orange (Citrus sinensis L. Osbeck) tree were excised and placed in a zip-lock bag. Eight additional random leaves were collected from the same tree and placed in a separate bag. The sampling process was repeated on six trees from the same grove. All samples were labeled and brought back to the laboratory.

From each of the six leaves (per one tree), four adjacent discs of tissue were excised along the midrib toward the petiole as illustrated in Fig. 1. For convenience, we designated these as positions one to four, one being the farthest from the petiole. All six discs from the same position for each tree were pooled and placed in a homogenizing tube for a total of four tubes per tree. The remaining eight leaves were randomly paired into four groups of two. From each midrib, $50 \mathrm{mg}$ of tissue were removed and combined into four samples of $100 \mathrm{mg}$ each. All samples were homogenized and titer determined by qPCR as described earlier. This experiment was repeated three times using different trees from the same grove (18 trees).

Test for possible time effect. From each tree in the variability test (18 trees), another set of six random symptomatic leaves were sampled at position 1, leaves were labeled and left on the tree. The disc samples were combined and placed in a homogenizing tube, brought to the laboratory, and frozen at $-20{ }^{\circ} \mathrm{C}$. This initial sample constitutes time $=$ 0 . At 7-d intervals, samples from subsequent positions (2, 3, and 4) were gathered and

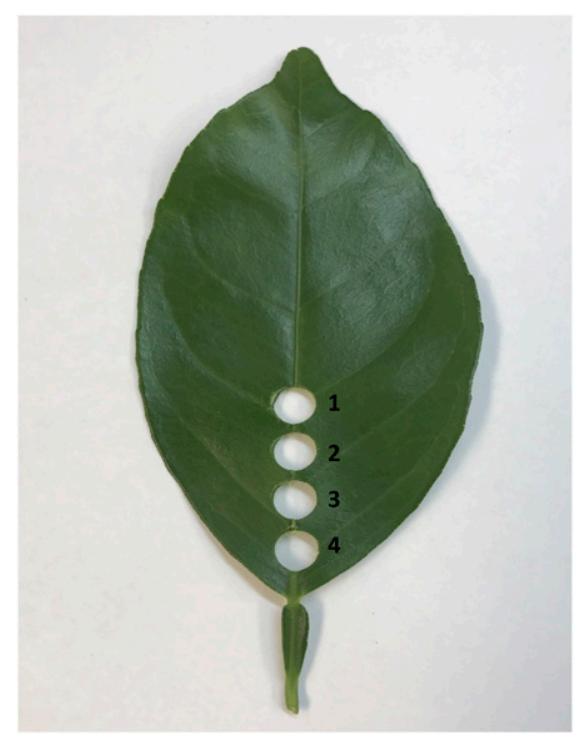

Fig. 1. Diagramatic depiction of a citrus leaf demonstrating the location of tissue excision. Discs were taken from the midrib to ensure consistent phloem tissue sampled throughout. First leaf disc sample was excised toward the center of the leaf, leaving sufficient space for the basipetal removal of subsequent disc samples. brought to the laboratory until all samples were obtained $21 \mathrm{~d}$ after the start of the experiment. At this time, all samples were homogenized, and titer was determined by qPCR as described earlier.

Effect of possible loss of leaves. Foreseeing the possible loss of leaves during a lengthy experiment, we designed a test to address this issue. From the same six trees, we excised six symptomatic leaves and brought them to the laboratory. A leaf disc was excised on position 1 for all six leaves, and discs were combined. To simulate the loss of one leaf, a second sample contained only five discs from five randomly selected leaves. Leaves continued to be selected randomly to collect the remaining discs in decreasing numerical order. Once a leaf had provided four leaf discs, it was removed from the pool. All samples were homogenized, and titer was determined by qPCR as already described. The experiment was repeated three times at different dates using the same set of trees as in experiment 1 .

Data analysis. The variability between samples was assessed by calculating the variance within natural groups. One such group is the variability within the four leaf discs from one tree. Doing this for all the data yields 18 variances. Another such group is the variability within the six leaf discs that are the first leaf punch from each leaf. Because there are four leaf discs and three fields, there are 12 variances. Finally, one can calculate the variance within the first leaf punch of the first tree in each grove. There are 24 of these values. Proc GLM in SAS 9.4 was used for analysis of variance (ANOVA). Multiple comparisons were done using the Tukey-Kramer test to control experimentwise error rate.

The approach to sample collection either as four discs taken ever closer to the petiole or as four leaf discs taken at 1-week intervals could result in a systematic shift in $\mathrm{Ct}$ values. Such a shift could increase variances, thereby producing a significant effect that is an artifact of sample collection. To address this issue, Proc GLM in SAS 9.4 was used to analyze the $\mathrm{Ct}$ values using ANOVA and regression. The primary issue is to know if the slope predicting $\mathrm{Ct}$ value in four leaf discs taken at one time differs from a similar procedure with leaf discs taken at 1-week intervals. The analysis of the experiment with different num- bers of discs used the same tools for data analysis.

\section{Results and Discussion}

Successful testing and development of new treatments to curb the effects of HLB in citrus trees rely on the accuracy in determining changes in the levels of infection. However, for citrus HLB, a main obstacle in measuring changes in foliar CLas titer is the considerable variability encountered within the tree canopy ( $\mathrm{Li}$ et al., 2009; McLean, 1970; Stover et al., 2010; Tatineni et al., 2008; Texeira et al., 2008). In addition, marked seasonal variations in CLas titer (Irey et al., 2011) introduce another element of inaccuracy. Given that leaf samples are collected and destroyed during the extraction process, a whole new set of random samples further decreases the degree of confidence needed to demonstrate statistical validity for any given treatment. The experiments described in this communication are aimed at supporting the development of a more accurate and simple method for the determination of CLas titer in studies where time is an integral factor of the experimental design. To achieve our goals, we tested adjacent fragments (punch-hole discs) of the same leaves by qPCR, and compared the $\mathrm{Ct}$ mean and variance to values obtained for randomly selected leaves. It should be noted that $\mathrm{Ct}$ numerical values are not based on a centesimal (1-100) scale but instead on a 20 -unit range (between 15 and 35 qPCR units for HLB), making numerical differences appear smaller. Also, Ct values are on a log scale, or powers of 2. A change in $\mathrm{Ct}$ value of 3.32 is equivalent to a 10-fold change in CLas DNA copies. If there is only one CLas DNA copy sequence per bacterium, then this is also a 10fold change in the number of bacteria.

Central to this study was determining whether the distinct methods of leaf tissue collection are fundamentally different in terms of statistical accuracy. To verify whether the variability in $\mathrm{Ct}$ values between subsamples from the same leaves is significantly smaller than that of random leaves, we analyzed the variance in the data. Table 1 lists the complete analysis of the variance in $\mathrm{Ct}$ values for both leaves and leaf discs. The variance in $\mathrm{Ct}$ values for the four leaf-disc positions within a single leaf (mean variance $=$ 0.21 ) was significantly lower than the variance of the four random leaf samples (mean

Table 1. Analysis of the variance for leaves and for leaf discs. Both the leaf disc method and random leaf samples consisted of pooling selected leaves from the same trees. "Leaves" means an estimate of the average variance of all leaves from a tree in a plot. Disc sampling started with the most apical disc locations, moving basally for subsequent samples (Fig. 1).

\begin{tabular}{llrccclc}
\hline & Source & df & Sum of squares & $F$ value & $\operatorname{Pr}>F$ & & Mean variance \\
\hline Leaves & Model & 2 & 9.12 & 1.3 & 0.2815 & Plots & $2.70 \mathrm{~A}$ \\
& Error & 51 & 178.92 & & & Trees & $2.76 \mathrm{~A}$ \\
& Total & 53 & 188.04 & & & Leaves & $1.85 \mathrm{~A}$ \\
Leaf discs & Source & df & Sum of squares & $F$ value & $\operatorname{Pr}>F$ & & Variance \\
\hline & Model & 2 & 18.69 & 6.08 & 0.0043 & Plots & $1.512 \mathrm{~A}$ \\
& Error & 51 & 78.38 & & & Trees & $1.30 \mathrm{AB}$ \\
& Total & 53 & 97.06 & & & Discs & $0.21 \mathrm{~B}$ \\
\hline
\end{tabular}


Table 2. Regression analysis of the effect of leaf position as related to disc sampling. Position 1 is farthest from the petiole along the midrib, and subsequent positions $(2,3$, and 4) are equally spaced and sequentially closer to the petiole. Tissue samples for each position consisted of six pooled discs from different leaves all taken at the same time.

\begin{tabular}{lcccc}
\hline Source & df & Sum of squares & $F$ value & $\operatorname{Pr}>F$ \\
\hline Model & 18 & 99.4 & 43.56 & $<0.0001$ \\
Error & 53 & 6.7 & $R^{2}=$ & 0.94 \\
Total & 71 & 106.1 & $F$ value & $\operatorname{Pr}>F$ \\
Source & df & Type III sum of squares & 90.41 & $<0.0001$ \\
\hline Plot & 2 & 22.9 & 41.70 & $<0.0001$ \\
Tree2 & 5 & 26.4 & 35.83 & $<0.0001$ \\
Plot $*$ Tree2 & 10 & 45.4 & 36.45 & $<0.0001$ \\
Leaf Discs & 1 & 4.6 & & $\operatorname{Pr}>|\mathrm{t}|$ \\
& & Slope (SE) & $<0.0001$ \\
\hline Leaf discs & & $0.23(0.038)$ & &
\end{tabular}

Table 3. Analysis of the variance for cycle threshold values between the four leaf-disks positions as a function of time, tree and block. Both set of disc samples (those analyzed at once and those at one-week interval) were taken from laves belonging to the same trees. Disc sampling started with the most apical disc locations and moving basally for subsequent samples (Fig. 1). Each sample was taken at 1-week intervals and compared with samples taken all at once.

\begin{tabular}{lcccc}
\hline Source & df & Sum of squares & $F$ value & $\operatorname{Pr}>F$ \\
\hline Model & 15 & 7.79 & 2.98 & 0.012 \\
Error & 20 & 3.48 & $R^{2}=$ & 0.69 \\
Corrected total & 35 & 11.27 & $F$ value & $\operatorname{Pr}>F$ \\
Source & df & Type III sum of squares & 1.71 & 0.2066 \\
\hline Block & 2 & 0.59 & 7.97 & 0.0105 \\
Method & 1 & 1.39 & 2.39 & 0.0749 \\
Tree & 5 & 2.08 & 1.88 & 0.1782 \\
Block * method & 2 & 0.66 & 3.53 & 0.0188 \\
Time * method & 5 & 3.08 & Average (variance) & \\
& & Method & 0.60 & A \\
\hline
\end{tabular}

Table 4. Regression analysis on the effect of time on the cycle threshold value of sampled leaf discs. Position 1 is farthest from the petiole along the midrib, and subsequent positions $(2,3$, and 4$)$ are equally spaced and sequentially closer to the petiole. However, unlike in Table 2, position 2 was sampled a week after position 1, position 3 a week after that, and position 4 a week after position 3 . Tissue samples for each position consisted of six pooled discs from different leaves remaining attached to the tree.

\begin{tabular}{lrcrr}
\hline Source & df & Sum of squares & $F$ value & $\operatorname{Pr}>F$ \\
\hline Model & 18 & 650.4 & 65.31 & $<0.0001$ \\
Error & 53 & 29.3 & $R^{2}=$ & 0.96 \\
Corrected total & 71 & 679.7 & $F$ value & $\operatorname{Pr}>F$ \\
Source & df & Type III sum of squares & 255.65 & $<0.0001$ \\
\hline Block & 2 & 282.9 & 75.03 & $<0.0001$ \\
Tree & 5 & 207.6 & 28.33 & $<0.0001$ \\
Block * tree & 10 & 156.7 & 5.81 & 0.0194 \\
Position & 1 & 3.2 & & $\operatorname{Pr}>|\mathrm{t}|$ \\
& & Slope (SE) & & 0.0194 \\
\hline Leaf discs & & $-0.20(0.078)$ &
\end{tabular}

Table 5. Mean of the variance in cycle threshold values for samples with a different number of leaf discs in each sample. All leaf samples containing different number of discs were taken from the same six leaves.

\begin{tabular}{lcccc}
\hline Source & df & Sum of squares & $F$ value & $\operatorname{Pr}>F$ \\
\hline Model & 5 & 124.3 & 7.48 & 0.0021 \\
Error & 12 & 39.9 & $R^{2}=$ & 0.76 \\
Corrected Total & 17 & 164.2 & \\
Punches & & Average (variance) & \\
\hline 1 & & $5.313 \mathrm{~A}$ & \\
2 & & $7.315 \mathrm{~A}$ & \\
3 & & $1.593 \mathrm{~B}$ & \\
4 & $0.561 \mathrm{~B}$ & \\
6 & & $0.644 \mathrm{~B}$ & \\
\hline
\end{tabular}

variance $=1.85$ ). Therefore, the two methods of leaf tissue sampling for CLas analysis are statistically different in their levels of accuracy and variability, with the leaf disc method being significantly more accurate. These results validate our hypothesis that the consistency between samples is significantly improved when adjacent tissue disc samples from the same leaf are used instead of random leaf sampling.

The data also demonstrated that there was a significant trend in the $\mathrm{Ct}$ values based on the position of the leaf disk (Table 2; $P<$ $0.0001)$, where each shift in position results in an increase of $0.23 \mathrm{Ct}$ units. The mean $\mathrm{Ct}$ value increased from 27.04 for position 1 to 27.70 for position 4 indicating a slight decrease in CLas population. This relatively small but significant decrease in $\mathrm{Ct}$ value toward the leaf apex is likely due to the accumulation of CLas in the acropetal direction (Fig. 1) during leaf expansion. This shift $(0.23$; Table 3$)$, however, is significantly smaller than the average deviation between random leaves (1.85; Table 1$)$.

For time-dependent experiments, it is pertinent to know whether removing a piece of leaf tissue triggers a physiological response that could affect CLas population and consequently its titer. This possibility was tested in a time course experiment where adjacent leaf disc samples were collected at 1 -week intervals starting from position 1 , and the average difference between two positions is summarized in Table 3 . The mean variance for leaf disks taken at 1-week intervals was 0.60 (Table 3), whereas if all done at the same time, the variance was 0.21 (Tables 1 and 3 ). Whereas the variance of the leaf samples taken over time (0.60; Table 3$)$ was higher than that taken at the same time, it remained significantly smaller than that using random leaf sampling (1.85; Table 1$)$. In addition, $\mathrm{Ct}$ values were examined for trends. The data showed a slight but significant effect of leaf position. The trend was smaller and of opposite sign $(+0.20$; Table 4$)$ relative to those sampled at the same time $(-0.23$; Table 2). These results indicated that an experimental design to monitor the effects of a given treatment on HLB-affected citrus trees based on leaf-punch samples from the same leaves will be more accurate than random selection of leaves due to the significantly smaller background variability in $\mathrm{Ct}$ values. Accuracy can be further amplified by running parallel-untreated controls to account for such treatment-induced fluctuations in CLas titer.

Foreseeing the possible loss of leaves during a lengthy experiment, we designed a test to determine the number of sampled leaves that could be lost before the variability in qPCR Ct values increases to the levels of random leaf sampling. Table 5 describes the statistical analysis of the experiment where we simulated the loss of up to five leaves. On the basis of the comparison of $\mathrm{qPCR} \mathrm{Ct}$ values between samples with increasing number of missing leaf discs and the control six leaves, the average variance is not statistically different with the loss of up to three 
leaves (Table 5). Given these results, it was estimated that up to three leaves could be lost through the course of the experiment without the loss of accuracy. With increasing loss of leaves, accuracy declines, and the benefits of the punch hole procedure is lost.

Studies on the development of new treatments against HLB (and other phloem bacterial diseases of trees) are plagued by the enormous variability in testing based on random leaf sampling compounded by the uneven distribution of CLas within individual trees. In the present report, we present data in support of a more accurate system to track bacterial populations (via $\mathrm{Ct}$ values) in citrus trees. This conclusion is based on the following sets of data: 1) Sampling adjacent portions of the same leaves significantly minimizes variability compared with sampling random leaves on the same tree (Table 1). 2) The system can be used effectively to track $\mathrm{Ct}$ changes for at least 6 weeks (Table 3) and likely longer if nontreated controls are run in parallel. 3) The accuracy is not significantly diminished by the loss of $50 \%$ of the sampled leaves (Table 5). Although designed to provide a more accurate method to determine HLB infection in citrus trees, the system is also applicable to other studies in other species (e.g., nutrition analysis, chlorophyll content) where leaf tissue samples are analyzed in a time-dependent factor.

\section{Literature Cited}

Ananthakrishnan, G., N. Choudhary, A. Roy, V.G. Sengoda, E. Postnikova, J.S. Hartung, A.L. Stone, V.D. Damsteeg, W.L. Schneider, J.E. Munyaneza, and R.H. Brlansky. 2013. Development of primers and probes for genus and species specific detection of 'Candidatus Liberibacter Species' by Real-Time PCR. Plant Dis. 97:1235-1243.

Aritua, V., D. Achor, F.G. Gmitter, L.G. Albrigo, and N. Wang. 2013. Transcriptional and mi- croscopic analysis of citrus stem and root response to Candidatus Liberibacter asiaticus infection. PLoS One, doi: 10.1371/journal.pone. 0073742.

Etxeberria, E., P. Gonzalez, D. Achor, and G. Albrigo. 2009. Anatomical distribution of abnormally high levels of starch in HLB-affected Valencia orange trees. Physiol. Mol. Plant Pathol. 74:76-83.

Gottwald, T.R. 2010. Current epidemiological understanding of citrus huanglongbing. Annu. Rev. Phytopathol. 48:119-139.

Graham, J.H., W.O. Dawson, and C. Robertson. 2008. Imidacloprid-induced systemic acquired resistance (SAR) in Cleopatra mandarin and development of HLB, p. 352. Proc. Int. Res. Conf. Huanglongbing, Orlando, FL. <https:// www.plantmanagementnetwork.org/proceedings/ irchlb/2008/presentations/IRCHLB.12.7.pdf>.

Halbert, S.E. and K.L. Manjunath. 2004. Asian citrus psyllids (Sternorrhyncha: Psyllidae) and greening disease of citrus: A literature review and assessment of risk in Florida. Fla. Entomol. $87: 330-353$.

Irey, M.S., T. Gast, J. Cote, P. Gadea, O. Santiago, L. Briefman, and J.H. Graham. 2011. Seasonal variability in HLB testing data in plant and psyllid samples in Florida, p. 72. In J.K. Burns, J.H. Graham, and T. Gottwald (eds.). Intl. Res. Conf. Huanglongbing, Orlando, FL. <https:// www.plantmanagementnetwork.org/proceedings/ irchlb/2011/presentations/IRCHLB_2011_4.6.pdf $>$.

Irey, M., T. Gast, and T.R. Gottwald. 2006. Comparison of visual assessment and polymerase chain reaction assay testing to estimate the incidence of the huanglongbing pathogen in commercial Florida citrus. Proc. Annu. Meet. Fla. State Hort. Soc. 119:89-93.

Li, W., J.S. Hartung, and L. Levy. 2007. Evaluation of DNA amplification methods for improved detection of "Candidatus Liberibacter species" associated with citrus huanglongbing. Plant Dis. 91:51-58.

Li, W., L. Levy, and J.S. Hartung. 2009. Quantitative distribution of 'Candidatus Liberibacter asiaticus' in citrus plants with citrus Huanglongbing. Bacteriology 99:139-144.

Louzada, E.S., O.E. Vazquez, W.E. Braswell, G. Yanev, M. Devanabiona, and M. Kunta. 2016. Distribution of 'Candidatus Liberibacter asiat- icus' above and below ground in Texas. Phytopathology 106:702-709.

McLean, A.P.D. 1970. Greening disease of sweet orange: Its transmission on propagative parts and distribution in partially diseased trees. Phytophylactica 2:263-268.

Roberts, P., R. Rouse, S.S. Teems, R.E. Sytsma, and R.E. Shobert. 2014. The effect of nutritional spray programs to mitigate symptoms of huanglongbing on fruit drop caused by HLB and citrus canker on Hamlin oranges. J. Citrus Pathol. 1:204.

Stover, E. and G. McCollum. 2011. Incidence and severity of Huanglongbing and Candidatus Liberibacter asiaticus titer among field-infected citrus cultivars. HortScience 46:1344-1348.

Stover, E., R. Shatters, Jr., G. McCollum, D. Hall, and Y. Duan. 2010. Evaluation of Candidatus Liberibacter asiaticus titer in field-affected trifoliate cultivars: Preliminary evidence for HLB resistance. Proc. Annu. Meet. Fla. State Hort. Soc. 123:115-117.

Tatineni, S., U. Shankar, S. Gowda, C.J. Robertson, W.O. Dawson, T. Iwanami, and N. Wang. 2008. In planta distribution of Candidatus Liberibacter asiaticus as revealed by polymerase chain reaction (PCR) and real-time PCR. Bacteriology 98:592-599.

Texeira, D.C., C. Saillard, C. Coutre, E.C. Martins, N.A. Wulff, S. Eviellard-Jagoueix, P.T. Yamamoto, A.J. Ayres, and J.M. Bové. 2008. Distribution and quantification of Candidatus Liberibacter americanus, agent of Huanglongbing disease of citrus in São Paulo, Brazil, in leaves of an affected sweet orange tree as determined by PCR. Mol. Cell. Probes 22:139-150.

U.S. Department of Agriculture. 2018. National Agricultural Statistics Service, Florida Citrus Statistics. <https://www.nass.usda.gov/Statistics_ by_State/Florida/Publications/Citrus/Citrus_ Statistics/index.php>.

Zhang, M., Y. Guo, C.A. Powell, M.S. Doud, C. Yang, and Y. Duan. 2014. Effective antibiotics against 'Candidatus Liberibacter asiaticus' in HLB-affected citrus plants identified via the graftbased evaluation. PLoS One, doi: 10.1371/journal. pone. 0111032 . 